\title{
Ligament Balancing in TKA. Is Intraoperative Strain Measurement of Ligaments Needed?
}

\author{
Hendrik P Delport* \\ University Hospital KU Leuven Orthopedics and Traumatology, Belgium
}

*Corresponding author: Hendrik P Delport, University Hospital KU Leuven Orthopedics and Traumatology August de Boeckstraat 1/8 9100 Sint-Niklaas, Belgium

\author{
ARTICLE INFO \\ Received: May 13, 2020 \\ Published: 幽 May 21, 2020 \\ Citation: Hendrik P Delport. Ligament \\ Balancing in TKA. Is Intraoperative Strain \\ Measurement of Ligaments Needed? . Bi- \\ omed J Sci \& Tech Res 27(5)-2020. BJSTR. \\ MS.ID.004562.
}

Abstract

Keywords: Total Knee Arthroplasty; Ligament Balancing; Ligament Strain

\section{Opinion}

Mechanical malalignment and component malpositioning have been identified as influencing factors for unsatisfactory clinical outcome. Although the impact of neutral mechanical alignment on implant longevity is still a matter of discussion, there is currently no better parameter to aim for when performing TKA. Soft tissues in human joints such as ligaments, tendons and the capsule both serve a passive mechanical (providing joint stability and determining joint kinematics) as well as a sensorial function. Up to now, mainly their mechanical function has been considered during surgical procedures (e.g. while balancing the knee joint during TKA). Recently, however, more and more orthopaedic surgeons experience that this may not be sufficient for clinical success. Despite the use of ever improved implant designs and more precise surgical techniques, a considerable number of patients (particularly after TKA) remains dissatisfied and suffers from reduced function compared to their healthy peers. This is an indication that the orthopaedics community is missing some important aspect of the working of joints. We believe that this may be the sensorial role of the soft tissues, particularly the ligaments [1].

Although it is not yet known exactly how ligaments sense deformation and strain, it is clear that they are capable of detecting quite small changes in length and reacting to those by influencing muscle tone. The relatively coarse joint balancing techniques which are in use during orthopaedic surgery are most probably not sufficient to defer to this delicate sensorial function in many patients. Therefore, we need a direct and accurate measurement of length changes of ligaments during surgery in combination with a way to tune implant alignment and/or perform ligament release to keep these length changes within acceptable limits. Some devices to improve knee joint balancing are already on the market, but they rely on indirect measurements (e.g. Verasense by Orthosensor or eLIBRA by Zimmer-Biomet measure contact pressures) and they provide only limited advice to the surgeon on how to change component alignment or perform ligament releases. We think that this approach is not sufficient. In fact, when looking at the philosophy behind these tools, it becomes clear that the providers mainly focus on the passive mechanical role of the soft tissues and disregard their sensorial function entirely [2].

An intra-operative sensor device that measures length changes in the medial collateral ligament directly is needed. A demonstrator has already been evaluated during a series of cadaver tests and has been shown to be easy to use as well as quite accurate, precise and reliable. In principle, it can also be used to perform measurements on the lateral collateral ligament, but due to surgical constraints 
during TKA, it will not be used for this in practice. To use the device to its full potential it is also necessary to develop an algorithm which will incorporate all measured data (during both laxity testing as well as flexion-extension cycles) and provide the surgeon with a suggestion on how to adapt tibial and/or femoral bone cuts and component alignment since we want to avoid ligament releases. This will require more elaborated tests on cadaver specimens (including also loaded joint motion using knee simulators) combined with specimen specific computer simulations.

Clinically, it will probably be necessary to combine the use of this type of sensor with intraoperative navigation and robotics. Indeed, information on ligament length changes, however accurate, will not be sufficient if it is not accompanied by information on how the joint is moving or changing position during intraoperative testing. This information can be provided by navigation systems. Furthermore, knowing how to change cuts or component alignment is one thing. Being able to execute the necessary changes with sufficient accuracy is quite another. For this, most probably robotic assistance will be needed.

ISSN: 2574-1241

DOI: $10.26717 / B J S T R .2020 .27 .004562$

Hendrik P Delport. Biomed J Sci \& Tech Res

(C) (P) This work is licensed under Creative Commons Attribution 4.0 License

Submission Link: https://biomedres.us/submit-manuscript.php

\section{Conclusion}

It is true that surgical robots will benefit from knowledge about ligament deformation, combined with such an algorithm. Indeed, what use is it to have a very accurate robotic system for orthopaedic surgery at one's disposal, if one doesn't have the necessary information to really take advantage of that increased accuracy? Conflict of Interest I declare that any economic interest or any conflict of interest does not exist.

\section{References}

1. Hendrik Delport, Luc Labey, Ronny De Corte, Bernardo Innocenti, Jos Vander Sloten, et al. (2013) Collateral ligament strains during knee joint laxity evaluation before and after TKA. Clinical Biomechanics 28(7): 777-782.

2. Delport H, Labey L, Innocenti B, De Corte R, Vander Sloten J, et al. (2015) Restoration of constitutional alignment in TKA leads to more physiological strains in the collateral ligaments. Knee Surg Sports TraumatolArthrosc 23: 2159-2169.

$\begin{array}{ll}\text { BIOMEDICAL } & \text { Assets of Publishing with us } \\ \text { RESEARCHES } & \text { - Global archiving of articles } \\ \text { - Immediate, unrestricted online access } & \text { - Rigorous Peer Review Process } \\ & \text { - Authors Retain Copyrights } \\ \end{array}$

